\title{
How to Increase Utilization and Dissemination of Evaluation Research Findings
}

\author{
Saurabh $^{1 *}$, Anjana ${ }^{2}$, Swati $^{3}$, Anand Singh ${ }^{1}$, S.K. Dubey ${ }^{4}$ and U.S. Gautam ${ }^{4}$ \\ ${ }^{1}$ KVK-II, Sitapur, India \\ ${ }^{2}$ CPS (P.G.) College, Moradabad, India \\ ${ }^{3} \mathrm{KVK}$, Pratapgarh, India \\ ${ }^{4}$ ICAR-ATARI, Zone-III, Kanpur, India \\ *Corresponding author
}

\section{A B S T R A C T}

\section{Keywords \\ Evaluation research, \\ Result dissemination and utilization \\ Article Info \\ Accepted: \\ 18 August 2018 \\ Available Online: \\ 10 September 2018}

The ratio of final dissemination and utilization of the evaluation based extension researches conducted in the field is very low in comparison to the experiment based researches. Even when with its long-term funding, strong coordination, and close links with actual practitioners or beneficiaries, the agricultural extension system has proved to be much less effective when the research-based outcomes to be disseminated stray from agricultural production technology into areas calling for attitudinal or behavioral changes. There are various causes and lacunas which need to be addressed in order to make evaluation researches more and more relevant and significant for the benefits of the society.

\section{Introduction}

"Research utilization fosters movement from innovation into practice"

Ultimately, for research to be relevant it must be linked to practice. If research results are not easily accessible and usable by those who need them most (i.e., persons with disabilities, their families, service providers, advocates, and other researchers, among others), they are of limited practical use. Dissemination speaks primarily to the process of knowledge transfer - the who, what, when, and how of moving ideas and information from the source(s) to intended recipient(s). Whereas uutilization speaks primarily to purpose and to impactwhy you want people to get the research outcomes you're putting forth, what use you want people to make of the ideas, information, or products, and how people are actually using them.

\section{Difference between dissemination and utilization}

Dissemination is the important item on most people's agendas: how to get research results to intended audiences in the most effective, cost-efficient manner. But utilization is a 
critical element in increasing the effective reach of research outcomes. Focusing only on the "D" in D\&U is like dialing nine numbers of a ten-digit telephone number: You may be 90 percent finished, but unless you dial that last digit, you'll never make the intended connection.

\section{All utilization is not the same}

The phenomenon of "utilization" varies from person to person. Even groups which may have been previously considered homogeneous, such as people with disabilities, share unique beliefs, abilities, and understandings. Strategies for achieving research utilization among such groups must embrace varying preferences and aptitudes for processing information about a new research outcome/application and rejecting it as unnecessary, as well as processing information about a new research-based innovation and applying it routinely in activities of daily living. Both of these outcomes are reflective of the wide continuum of utilization.

\section{Difficulties in research utilization}

The purpose of research is to be of use-to change current practice, or to confirm it. Yet the process of moving new understandings and new products from research to practice usually takes years, decades, or even generations. Although there are good reasons for moving carefully-new research needs to be evaluated, replicated, and refined-too often the pace of change is set, not by a rigorous process of review and refinement, but by the gap between the research community and the world of practice.

Research on dissemination, or knowledge utilization as it is sometimes called, has yielded a wealth of information about what does and does not work. But, due to this gap, those understandings for the most part have not moved from the research communitythose who study the process of knowledge use - to the practice community-those responsible for adopting and applying research outcomes. As a result, most dissemination practices are still based on a mechanistic, linear conception of dissemination as a process of "getting the word out."

Approaches designed to promote knowledge utilization within the fields of rehabilitation and education traditionally have been drawn from the agricultural extension model, whose basic presumption is that people will use research-based products only if they have access to information about them. The success of the agricultural extension model, along with other experience, tells us that this presumption is true, in some cases, and under specific circumstances. However, even with its longterm funding, strong coordination, and close links with practitioners, the agricultural extension system has proved to be much less effective when the research-based outcomes to be disseminated stray from agricultural production technology into areas calling for attitudinal or behavioral changes.

\section{Low utilization of findings}

Major disappointment in the field of evaluation research is general recognition of low utilization of findings. Researchers tend to cite the organizational and professional resistances to change as well as the political consideration also seems to dominate planning and policy making. Administrators and policy makers express dissatisfaction with:

Poor research methodology

Irrelevant and inappropriate studies

Lack of comparable studies

No cumulative body of evidences 
Lack of clear direction or recommendations emanating from the research

Poor interaction between administrators and policy makers

Poor communication between them

Lack of involvement of administrators and policy makers in the planning and conduct of the study

Poor timing of report and inadequate dissemination

These factors required close attention to improve the likelihood of utilization

\section{Constructivist learning theory and utilization of research results}

Current perspectives on knowledge utilization draw from a theory of learning known as constructivism. According to constructivist principles, knowledge is not a "thing"-a static, inert object to be sent and received. Rather, knowledge is a fluid set of understandings shaped both by those who originate it and by those who use it. "This casts the user as an active problem-solver and a constructor of his or her own knowledge, rather than as a more passive receptacle of information and expertise"(Hutchinson and Huberman, 1993). This implies that utilization is most likely to occur when potential users determine that they have a need for particular information. This constructivist orientation is the basis for modern day marketing research and associated techniques designed to influence a user's perception of "need," and similarly, utilization models must recognize "need" as a powerful force in producing and shaping outcomes.

Past and faulty "models of knowledge utilization" have been based upon a variety of beliefs about how learning takes place. For example one "model" suggested the human mind was a tabula rasa, or a blank slate to be written upon. This knowledge utilization model also has been called "the bucket theory of the mind" (Buchman, 1982), in which the brain was viewed as an empty vessel into which knowledge was poured.

Another common and, again, faulty "utilization model" was the notion that people acted as sponges, "soaking up" knowledge, a role that is somewhat more active than that of an empty vessel. However, this model fails as well as the learner is considered to absorb knowledge taken in wholesale, without filtering or processing. Yet another notion often used in this era of technology is that of the brain as a computer, which processes in an orderly, systematic fashion the information that is received from outside sources.

None of these "models" adequately describes the ways in which learners process information. New knowledge is not merely filtered and sorted, but transformed by the learner's pre-existing experiences and understandings. This conceptual framework suggests the building and shaping of new structures as a part of the knowledge utilization process.

\section{Research findings utilization model}

Utilization models must incorporate intended users' needs, contexts, and readiness for change for application of the new researchbased knowledge to occur. The degree of detail in this portion of a utilization model rests upon the level of detailed understanding about the intended user group(s) and the nature of the research result being disseminated for use. The level of diversity and options in a utilization model depends upon the extent of diversity within a particular target audience. A successful utilization model 
must rely on effective and well-defined interactions between basic process elements of the potential user, the content of the message, the context required to obtain the message, the medium of the message, and the source of the message/research result(s). Figure 1 provides a diagram of the interaction among these elements.

\section{Building blocks of utilization modeling}

To develop the knowledge transfer base that underlies utilization, there are five common core elements that are keys to success. These core elements strongly influence whether utilization efforts will be effective:

Source - Where does the research information come from?

Content - What is the research information about?

Context - How does the research information relate to existing knowledge or products?

Medium - How can I get the research information?

User - How can I benefit from this research information?

\section{Media used for research results utilization}

There are various media from which researchers can disseminate the findings of the research:

Workshops, conferences, symposiums and seminars

Newspaper, journal, magazine articles etc.

Public service announcements (films etc.)

Web sites
Report of the researches to the person concerned etc.

\section{Implications for dissemination and utilization efforts}

What does all this mean for those whose responsibilities include dissemination for the purpose of utilization of research outcomes? It means, first, that dissemination is not synonymous with publication. Merely creating documents or innovations and sending out information, whether via an article in a scholarly journal or the World Wide Web, will not create a "utilization model." This type of model must be developed around the intended users - their worries, beliefs, constraints, and priorities, and the people and organizations whose opinions they tend to value.

Utilization models must incorporate intended users' needs, contexts, and readiness for change for application of the new researchbased knowledge to occur. The degree of detail in this portion of a utilization model rests upon the level of detailed understanding about the intended user group(s) and the nature of the research result being disseminated for use. The level of diversity and options in a utilization model depends upon the extent of diversity within a particular target audience.

\section{Considerations for utilization model}

Developing new utilization models takes some careful thought about matching a given strategy with a specific desired outcome. A wide array of "variables" must be accounted for in this type of modeling process. For example, a utilization model must specify:

An action that will link a specific user(s) with a specific message/research result; specific, accessible format and a respected information/research source. 


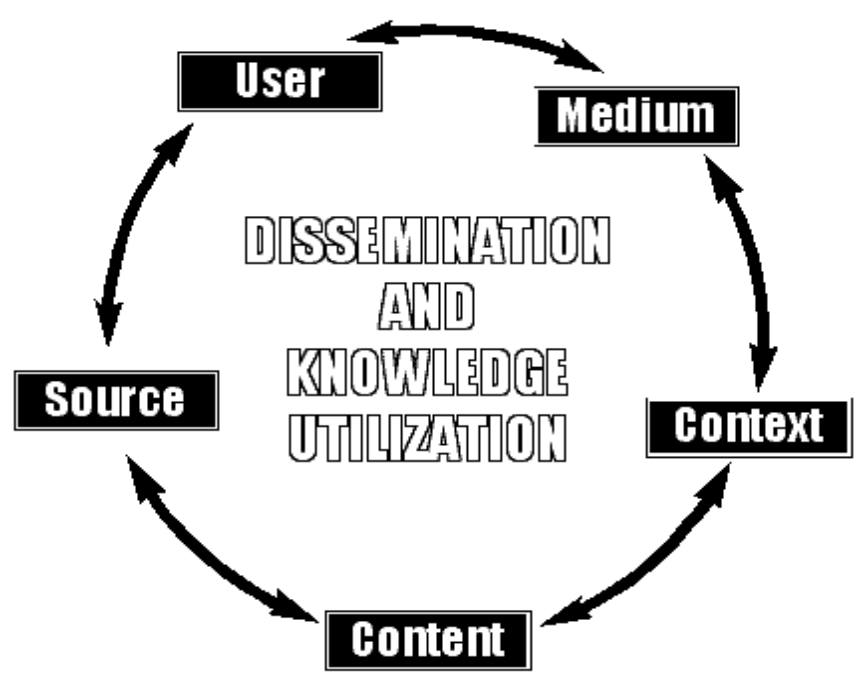

Fig-1

This may sometimes be referred to as a public information campaign.

An action must be "matched" with known available resources required, such as staff time, budget, materials, equipment, and technology.

Elements of the activity or activities to be conducted, such as workshops, newspaper articles, public service announcements, web sites, or others should be designed for the individuals or groups each is intended to reach such as, for example, people with spinal cord injuries, family members of people with cognitive disabilities, independent living center program directors, or others.

Short-term outcomes can be planned as casual foundations for subsequent impacts.

For example, a short-term outcome could be increased awareness of the benefits of wearing a helmet while cycling, an intermediate outcome could be behavior change in the form of routinely wearing a helmet, and a long-term impact could be the reduction of traumatic brain injuries of cyclists.
Clearly, the skillful connection of knowledge transfer efforts with targeted utilization plans produces a well-conceived, integrated approach to moving research into practice.

\section{Effective dissemination and utilization of results}

Both dissemination and utilization activities must be planned and conceived to meet the needs of a specific user if each is to be efficient and ultimately successful. Utilization that may occur through activities that are not structured-for example, through dissemination activities-may just happen. This approach to utilization is not, however, desired for researchers to consistently use and assume will be effective in moving research to practice. A need exists for clear ways to link dissemination and utilization for the purpose of moving research findings into the hands and minds of those that can most benefit from them. Facilitating such utilization requires a structured, planned approach.

The research on utilization is quite clear: the meaning of research is conducted by the user. Individuals translate research findings 
through the lens of prior knowledge and understanding, making sense of new knowledge in the context of their daily activities. It is research on learning that is the foundation of understanding knowledge utilization. The promotion of utilization is as much more than distribution and offering access. It calls for two major changes in current utilization practices in the field of education: "First, we should focus more on the context of knowledge users, and second, we should strengthen the integration between research and dissemination."

\section{References}

Buchman, M. (1982). The use of knowledge: Conceptual problems and empirical confusion. Occasional Paper No. 57. East Lansing, MI: Institute for Research on Teaching.
Hutchinson, J., and Huberman, M. (1993). Knowledge dissemination and utilization in science and mathematics education: A literature review. Washington: National Science Foundation, NSF 93 75. http://www. researchutilization.org/cgibin/rushlinkm gr.cgi?url=http://www.nsf.gov/pubs/stis 1993/nsf9375/nsf9375.txt. cited on 10.04.2018

Literature Review on Dissemination and Utilization of Research Results, http://www.ncddr.org/products/research exchange. cited on 10.04.2018.

Rutman, L., Evaluation research methods, Sage Publications.

Welcome to Research Utilization, http:// www.researchutilization.org. Cited on 10.04.2018

\section{How to cite this article:}

Saurabh, Anjana, Swati, Anand Singh, S.K. Dubey and Gautam, U.S. 2018. How to Increase Utilization and Dissemination of Evaluation Research Findings. Int.J.Curr.Microbiol.App.Sci. 7(09): 2484-2489. doi: https://doi.org/10.20546/ijcmas.2018.709.308 